\title{
In vitro and in vivo Evaluation of Hemocompatibility of Silk Fibroin Based Artificial Vascular Grafts
}

\author{
Derya Aytemiz $^{1}$, Yu Suzuki ${ }^{1}$, Tomoko Shindo ${ }^{2}$, Toshiki Saotome ${ }^{1}$, Ryou Tanaka ${ }^{3} \&$ Tetsuo Asakura ${ }^{1}$ \\ ${ }^{1}$ Department of Biotechnology, Tokyo University of Agriculture and Technology, Nakacho, Koganei, Tokyo, \\ Japan \\ ${ }^{2}$ Hadano Research Institute, Food and Drug Safety Center, Ochi-ai, Hadano, Kanagawa, Japan \\ ${ }^{3}$ Division of Animal Life Science, Tokyo University of Agriculture and Technology, Harumicho, Fuchu, Tokyo, \\ Japan
}

Correspondence: Tetsuo Asakura, Department of Biotechnology, Tokyo University of Agriculture and Technology, 2-24-16 Nakacho, Koganei, Tokyo 184-8588, Japan. E-mail: asakura@cc.tuat.ac.jp

Received: December 4, 2013 Accepted: February 6, 2014 Online Published: March 6, 2014

doi:10.5539/ijc.v6n2p1

URL: http://dx.doi.org/10.5539/ijc.v6n2p1

\begin{abstract}
Artificial vascular grafts with low thrombogenicity are generally required to avoid blood platelet adhesion and to minimize intimal hyperplasia, thus retaining vascular patency. In this study, we aimed to determine the acute and subacute hemocompatibility of silk fibroin (SF) grafts by in vitro and in vivo evaluation. Blood contact reaction with SF grafts was examined by thrombin-anti-thrombin III complex (TAT) formation, platelet activation level by $\beta$-thromboglobulin ( $\beta$-TG), complement system response (C3a and SC5b-9), platelet and fibrin deposition and compared with commercially available polyethylene terephthalate (PET) artificial grafts in vitro. The biocompatibility and coagulation-inducing effect of coating materials were evaluated by in vivo implantation in rats. Two weeks after implantation, SF grafts showed low subacute coagulation. All blood parameters evaluated for animals implanted with SF-coated grafts showed almost the same values as those for sham-operated animals. Our results support the suggestion that SF will be a suitable material for vascular regeneration in future.
\end{abstract}

Keywords: silk fibroin, gelatine, polyethylene terephthalate, artificial vascular graft, hemocompatibility

\section{Introduction}

The increasing morbidity in modern society due to cardiovascular disease has made it an urgent necessity to develop blood vessel substitutes, especially those with an internal diameter of less than $6 \mathrm{~mm}$, to replace diseased coronary and below-the-knee vessels. For the last 50 years, therapies for such diseases have involved surgical replacement of the damaged vessels with autologous vessels or synthetic materials. Limited supply and metabolic diseases are problems for autologous vessel replacement, while acute thrombosis, infection and lack of biocompatibility are problems associated with the current synthetic materials (Murugesan et al., 2008). Laboratory models also have shown that synthetic grafts are substantially more thrombogenic compared to autologous veins. Prosthetic materials cause platelet adhesion and activation of the coagulation cascade on the graft (Heyligers et al., 2006). Nevertheless, polyester synthetic materials such as polyethylene terephthalate (PET) have been used routinely for surgical replacement of the large arteries with high blood flow velocity for the past half century (Devine et al., 2004). In order to design small-diameter arterial prosthesis, the complete design specifications should be determined based on the features of successful large diameter vascular prosthesis (How et al., 1992).

Bombyx mori silk fibres have been used as commercially available sutures since the end of the 19th century and have proved to be effective biomaterials (Vepari et al., 2007). Silk fibroin (SF) has been reported to have many inherently superior properties as a biomaterial, in terms of mechanical properties, environmental stability, biocompatibility, low immunogenicity and biodegradability (Vepari et al., 2007; Asakura et al., 1994; Demura et al., 1989, 1989a, 1991); therefore, many applications of SF have been studied and investigated as biomaterials substrate for cell growth related to revascularization.

In the last decade many studies have concentrated on $\mathrm{SF}$ application such as, manufacturing silk micro-tubes using the layer-by-layer deposition of concentrated SF solution (Lovett et al., 2007), gel spinning (Lovett et al., 
2008, 2010) and electrospining methods, electrospun silk matrices with vascular tissue engineering (Jin et al., 2004; Zhang et al., 2008; Soffer et al., 2008; Rockwood et al., 2008; Bondar et al., 2008; Gupta et al., 2010; Stoppato et al., 2013) and bilayered vascular graft from SF (Lovett et al., 2010). However, the burst strengths of those silk micro-tubes and electrosupun tubes were significantly low to compare with native vessels (Lovett et al., 2007). Meanwhile, the biological potential of electrospun silk matrices for vascular tissue engineering was evaluated by human aortic endothelial cells (HAECs) and human coronary artery smooth muscle cells (HCASMCs) (Zhang et al., 2008). Nevertheless, electrospun silk matrices have not been evaluated under blood stream in vivo. Recently, silk tubes produced by using an aqueous gel spinning technique (Lovett et al., 2010) were first assessed in vitro in terms of thrombogenicity (thrombin and fibrinogen adsorption, platelet adhesion) and vascular cell responses (EC and SMC attachment and proliferation) in comparison with polytetrafluoroethylene (PTFE), a synthetic material most frequently used for vascular grafts. Silk tubes were then implanted into the abdominal aortas of Sprague-Dawley rats. Over a 4-week period, gel spun grafts exhibited EC lining of the lumen surfaces; however, the evaluation period was kept preliminary. Comprehensive studies, both in vitro and in vivo, have demonstrated that silk fibroin is more biocompatible than other commonly used polymeric degradable biomaterials, such as PLA, PGA and collagen (Lovett et al., 2008). In addition SF have been applied as a coated material by impregnated into a porous knitted polyester (Dacron) graft (Huang et al., 2008). In contrast to collagen-coated grafts, the SF coated polyester grafts showed less thrombogenesis and induced host cell migration along the matrix without foreign body or inflammatory reactions. Moreover, it appears to encourage the development of endothelial-like cells (Huang et al., 2008).

In our previous study, endothelial and smooth muscle cells migrated into an SF graft early after implantation into rat abdominal aorta and organized into endothelial and medial layers (Enomoto et al., 2010). The SF graft showed higher patency than a PTFE graft of $1.5 \mathrm{~mm}$ inner diameter at the end of 1 year. These results suggest that $\mathrm{SF}$ is suitable for use as a vascular graft material with good biocompatibility in rat implantation study. Unfortunately rat studies were limited to observe human blood reaction with vascular graft substrate. There are few previous studies which SF has been investigated for blood compatibility; but those studies mainly concentrate on investigating SF material, as silk film form. The film shows perfectly smooth surface and therefore cannot be used as a coating form on vascular graft surface directly. The coating treatments are an important step in the fabrication of artificial blood vessels. Various materials such as gelatin, collagen and a heparinized hydrophilic material have been used to prevent blood leakage and to improve performance and provide good biocompatibility. Coating materials come into direct contact with blood when artificial blood vessels are implanted, and therefore, anti-thrombogenicity of the coating materials also affects the initial vessel patency.

In this study, we tested a fabricated vascular tube coated with SF by two different coating methods and by coating with gelatin. We examined the hemocompatibility of the SF vascular tube and the coating materials, compared it with that of a commercially available PET vascular tube using human blood. We also observed the activity of platelet and fibrin adhesion on the vascular tube surface by scanning electron microscopy (SEM). In addition, we conducted an in vivo rat implantation study to compare an SF vascular tube coated with SF by two different methods with an SF vascular tube coated with gelatin and evaluated both in comparison to sham operation. Biocompatibility and hemocompatibility of the coating materials were evaluated at 2 weeks after implantation by analysing rat whole blood. The coagulation effects of the coating materials on blood plasma were evaluated. Our results from in vitro and in vivo studies support the notion that SF has good hemocompatibility as a material for artificial vascular grafts.

\section{Materials and Methods}

\subsection{Preparation of SF Tubes}

Extracted SF from B. mori silkworm (Matsuda Shoji, Kyoto, Japan) and computer-controlled double-raschel knitting machine was used to prepare the knitted SF tubes as described previously (Aytemiz et al., 2012). The double-raschel SF tubes were then degummed in a solution of sodium carbonate $[0.08 \%(\mathrm{w} / \mathrm{v})]$ and Marseille soap $[0.12 \%(\mathrm{w} / \mathrm{v})]$ at $95^{\circ} \mathrm{C}$ for $120 \mathrm{~min}$ in order to remove the silk sericin before coating (Aytemiz et al., 2012). In this study, we used $6 \mathrm{~mm}$ inner diameter vascular tubes for in vitro evaluation and $1.5 \mathrm{~mm}$ inner diameter tubes for in vivo evaluation.

\subsection{Preparation of SF Aqueous Solution}

To coat the SF tubes, SF aqueous solution was prepared as described previously (Vepari et al., 2007; Demura et al., 1989). In brief, B. mori cocoons were placed in boiling water at $95^{\circ} \mathrm{C}$, after which the threads were reeled off. The dried silk threads were degummed in a solution of sodium carbonate $[0.08 \%(w / v)]$ and Marseille soap 
$[0.12 \%(\mathrm{w} / \mathrm{v})]$ at $95{ }^{\circ} \mathrm{C}$ for $120 \mathrm{~min}$ in order to remove silk sericin from the surface of the raw silk fibres. The degummed SF fibres were then dissolved in a $9 \mathrm{M}$ lithium bromide (Wako, Tokyo, Japan) solution to a concentration of $10 \%(\mathrm{w} / \mathrm{v})$ at $37^{\circ} \mathrm{C}$ for $1 \mathrm{~h}$ and then dialyzed against distilled water for 3 days at $4{ }^{\circ} \mathrm{C}$ using a cellulose membrane (MWCO 14,000) (Sanko Junyaku, Tokyo, Japan). The final concentration of the fibroin/water solution was 3-8\% (w/v). Freshly prepared SF aqueous solution was used to coat the SF tubes.

\subsection{Coating of SF Tubes With SF and Gelatin Aqueous Solution}

Two SF coating methods, lyophilization coating and sponge coating, were used. Lyophilization coating was performed as follows: $6 \mathrm{~mm}$ and $1.5 \mathrm{~mm}$ diameter SF tubes were mounted onto PTFE tubes and immersed in 5\% $\mathrm{w} / \mathrm{w} \mathrm{SF}$ aqueous solution and incubated under $100 \mathrm{hPa}$ for $30 \mathrm{~min}$. The silk fibroin based tube was then transferred to liquid nitrogen for $1 \mathrm{~min}$ and frozen at $-80^{\circ} \mathrm{C}$ for $1 \mathrm{~h}$. Following this step, grafts were lyophilized overnight and fixed in $70 \%$ ethanol for $10 \mathrm{~min}$. Next, the grafts were rinsed in distilled water and left to dry at room temperature for approximately $1 \mathrm{~h}$. The same procedure was repeated again and the grafts were then autoclaved at $120^{\circ} \mathrm{C}$ for $20 \mathrm{~min}$. The $\beta$-sheet rich conformation of the SF coating following these procedures was confirmed by FT-IR and solid state NMR. For sponge coating, poly(ethylene) glycol diglycidyl ether (PEGDE) (Sigma-Aldrich Co. LLC., MO, USA) was used as a porogen, as described previously (Aytemiz et al., 2012). In brief, $6 \mathrm{~mm}$ and $1.5 \mathrm{~mm}$ diameter SF tubes mounted onto PTFE tubes were placed into an acryl tube and an SF:PEGDE = 1:1 solution was injected between the SF tube and the acryl tube. Then, the tubes were frozen at $-20{ }^{\circ} \mathrm{C}$ overnight. Next day, grafts were transferred to distilled water and rinsed for 3 days to remove PEGDE and then autoclaved at $120^{\circ} \mathrm{C}$ for $20 \mathrm{~min}$.

The SF graft coated with gelatin was prepared as follows. Medical grade gelatin (Medigelatin, Nippi Incorporated, Tokyo, Japan) was dissolved in distilled water to prepare a $3 \% \mathrm{w} / \mathrm{v}$ gelatin aqueous solution. SF tubes of $6 \mathrm{~mm}$ and $1.5 \mathrm{~mm}$ diameter were mounted onto PTFE tubes and immersed in the gelatin solution for 30 min. To remove excess gelatin and create a uniform coating, the tubes were rolled under a spatula and then allowed to stand at $4{ }^{\circ} \mathrm{C}$ for $1 \mathrm{~h}$. Finally, the coated gelatin was crosslinked using $10 \%$ glutaraldehyde (Tokyo Chemical Industry Co., LTD., Japan). The same procedure was repeated and the grafts were then fixed in $70 \%$ ethanol for $30 \mathrm{~min}$. Finally, they were sterilized by autoclaving at $120^{\circ} \mathrm{C}$ for $20 \mathrm{~min}$.

The samples evaluated in this study are denoted as follows: lyophilized SF coating SF grafts (SF-LC), sponge coating SF grafts (SF-SC), gelatin coating SF grafts (SF-GC), and gelatin coating PET graft (PET-GC). PET-GC is a commercially available artificial vascular graft (Gelsoft巴 Plus; Terumo, Tokyo, Japan).

\subsection{In vitro Evaluation of Hemocompatibility With Human Whole Blood}

\subsubsection{Examination Conditions}

In this study, fresh human blood was used in experiments. The donors' approval for the provision of blood was obtained and confirmed by written consent. Fresh human blood was drawn from the brachial veins of three healthy, medication-free volunteers. A sample of $135 \mathrm{~mL}$ of blood was collected directly into a heparinized syringe (1 IU/mL heparin) (LEO Pharmaceutical Products Sarath Ltd., Denmark). Heparinized blood was stored at room temperature and used within $1 \mathrm{~h}$ of collection.

Evaluation of the interaction between grafts and blood was performed under two conditions, one static and the other under rotation using the Chandler loop method (Tepe et al., 2006). Each blood sample was initially studied before the period of exposure to the graft and each experiment included a blank $(n=3)$. For evaluation under static condition, a $15 \mathrm{~cm}$ length of a $0.8 \mathrm{~cm}$ internal and $1.4 \mathrm{~cm}$ outer diameter polypropylene-thermoplastic elastomer copolymer tube was used. The inner surface area of the tube was $37.7 \mathrm{~cm}^{2}$ and the interior volume was $7.5 \mathrm{~mL}$. The surface ratio of blood to tube surface area was calculated as $5.4 \mathrm{~cm}^{2} / \mathrm{mL}$, and $7 \mathrm{~mL}$ heparinized blood was placed into the tube, taking care to avoid contamination. For the static condition test, each graft was vertically cut into $1 \mathrm{~cm}$ lengths and washed with physiological saline solution. After this, each sample was placed into the static tube. The vascular tube surface area was $3.8 \mathrm{~cm}^{2}$, and the blood to surface area ratio on the vascular tube supplied was $0.54 \mathrm{~cm}^{2} / \mathrm{mL}$. Samples were incubated with heparinized blood at $37{ }^{\circ} \mathrm{C}$ for $2 \mathrm{~h}$. Evaluation under rotation condition was performed as follows. The diameter of the tube was the same as that used for static evaluation, and the tube length was $30 \mathrm{~cm}$ in circuit. The inner surface area of loops and the content volume were determined as $75.4 \mathrm{~cm}^{2}$ and $15.1 \mathrm{~mL}$, respectively. The ratio of blood to vascular tube surface area was calculated to be the same as that under static condition; $14 \mathrm{~mL}$ of blood was placed into a loop in $2 \mathrm{~cm}$ lengths to test each graft. The Chandler loop was rotated 30 times a min at $37^{\circ} \mathrm{C}$ for $1 \mathrm{~h}$.

\subsubsection{SEM Observations}

Grafts after in vitro evaluation were observed by SEM (S3500N, Hitachi, Tokyo, Japan) to visualize microscopic 
changes on the surfaces of the vascular tubes as well as fibrin deposition, platelet adhesion with formation of pseudopodia and platelet aggregation. Each graft specimen was rinsed with $0.01 \mathrm{M}$ phosphate-buffered saline (PBS) and then fixed with $0.1 \mathrm{M}$ phosphate-buffered $1.25 \%$ glutaraldehyde for $2 \mathrm{~h}$ under ice. After fixation, the samples were cut and post-fixed with similarly buffered $2 \%$ osmium tetroxide and then rinsed with PBS three times. Thereafter, the samples were treated by dehydration in ethanol, immersion in t-butanol and lyophilization; finally, the surface was sputter-coated with gold ion. The intensity of fibrinogen, platelet adhesion or aggregation was evaluated at random five different points for each blood sample. Results were classified as follows: (0) indicates no sign of fibrinogen, platelet adhesion or aggregation. (1), (2), (3) and (4) indicate very mild, mild, moderate and severe, respectively $(\mathrm{n}=3)$.

\subsubsection{Measurement of TAT, $\beta$-TG, C3a and SC5b-9}

We investigated thrombin activation by measuring levels of thrombin-anti-thrombin III complex (TAT), platelet activation level by $\beta$-thromboglobulin ( $\beta$-TG) assay and complement system response by C3a and SC5b-9 assay. For TAT evaluation, $1.8 \mathrm{~mL}$ heparinized blood was collected into a $3.8 \%$ sodium citrate-containing tube (Terumo, Tokyo, Japan) and allowed to stand on ice for $5 \mathrm{~min}$. For $\beta$-TG evaluation, $1.8 \mathrm{~mL}$ heparinized blood was collected into a tube containing $0.2 \mathrm{~mL}$ CTAD (BD, Franklin Lakes, NJ) and allowed to stand on ice for $15 \mathrm{~min}$. For C3a and SC5b-9 evaluation, $1 \mathrm{~mL}$ heparinized blood was collected into an EDTA-2K blood tube and mixed with $1 \mathrm{mg} / \mathrm{mL}$ Futhan (Torii Pharmaceutical Co., Ltd., Tokyo, Japan) and $0.1 \mathrm{~mL}$ physiologic saline solution. Levels of TAT, $\beta$-TG, C3a and SC5b-9 were determined in plasma samples obtained by centrifugation of the blood samples using Enzygnost ${ }^{\circledR}$ TAT micro (Siemens, Munich, Germany), $\beta$-TG TMB (Roche Diagnostics, Mannheim, Germany), the MicroVueTM C3a plus EIA kit and the MICROVUETM SC5b-9 plus EIA kit (QUIDEL, San Diego, CA), respectively.

\subsection{In vivo Evaluation on Rats}

Female Sprague-Dawley rats weighing 200-300 g were purchased from Charles River Laboratories, Yokohama, Japan. The total number of rats used in this study was 12 . All rats were kept in microisolator cages with a 12-h light/dark cycle. All experimental procedures and protocols were approved by the Animal Care and Use Committee of the University of Tokyo and complied with the Guide to the Care and Use of Laboratory Animals (Approval number: 23-84).

To investigate the biocompatibility and hemocompatibility of the coating materials, the SF grafts coated with SF-LC, SF-SC and SF-GC were implanted into the rat abdominal aorta, which was approximately $1.5 \mathrm{~mm}$ in internal diameter and $10 \mathrm{~mm}$ in length. In brief, the rats were anesthetized by intraperitoneal injection of pentobarbital $(50 \mathrm{mg} / \mathrm{kg}$ body weight). The abdominal aorta was exposed, and the aortic branches in this segment were ligated. After intravenous injection of heparin $(100 \mathrm{IU} / \mathrm{kg})$, the proximal and distal portions of the infrarenal aorta were clamped. The SF grafts $(1.5 \mathrm{~mm}$ in diameter and $10 \mathrm{~mm}$ in length) were replaced by end-to-end anastomosis using interrupted 9-0 monofilament nylon sutures (BEAR, Tokyo, Japan). The distal and proximal clamps were slowly removed, and blood flow was restored through the SF grafts. Sham operation as a negative control was performed in the same manner but without using any graft. After cutting the host abdominal arteries, the ends of the native artery were re-sutured together, and blood flow was restored $(n=3)$. Two weeks after graft implantation, rat whole blood was collected by venipuncture under pentobarbital anaesthesia and blood was collected in sterile tubes containing $0.5 \mathrm{~mL}$ EDTA and $1 \mathrm{~mL}$ sodium citrate. Blood cells were counted immediately in an automatic haematological analyser equipped with veterinary software (Nihon Kohden Corporation, Tokyo, Japan). Total peripheral white blood cells (WBCs; consisting of leucocytes, lymphocytes, monocytes and neutrophils) and procalcitonin (PCT) level were measured to evaluate biocompatibility, while platelet count (PLT) and platelet distribution width (PDW) were measured to evaluate hemocompatibility. Serum prothrombin time (PT) and activated partial thromboplastin time (APTT) were also measured using semiautomatic blood coagulation analysis equipment (COAG2V, Wako, Japan). In brief, approximately $1 \mathrm{~mL}$ sodium citrated blood was centrifuged immediately, and plasma was separated into a new tube. A $25-\mu \mathrm{L}$ aliquot of plasma was then applied to the reagent strip to measure PT or APTT.

\subsection{Statistical Analyses}

Values are shown as mean \pm standard deviation (SD). All groups were compared by one-way ANOVA. To identify differences between the groups, we used Bonferroni's and tukey's post-hoc multiple comparisons test. Data analyses were performed using the commercial statistical analysis software GraphPad Prism (Version 5.0a, GraphPad, San Diego, CA). A P level $<0.05$ was considered statistically significant. 


\section{Results}

\subsection{SEM Observations of Graft Surfaces}

Characterization of vascular graft without coating and after coating treatment by SEM image of low magnification are shown in Figure 1(a-f) in following order top-down, outer surface, inner surface and cross sections of graft walls, respectively. SEM images of SF and PET vascular grafts before coating treatment, are shown in Figures 1(a) and 1(e). After treatment of SF by lyophilization coating, sponge coating and gelatin coating are shown in Figures 1(b), 1(c) and 1(d), respectively. Figure 1(e) shows gelatin coated PET graft.

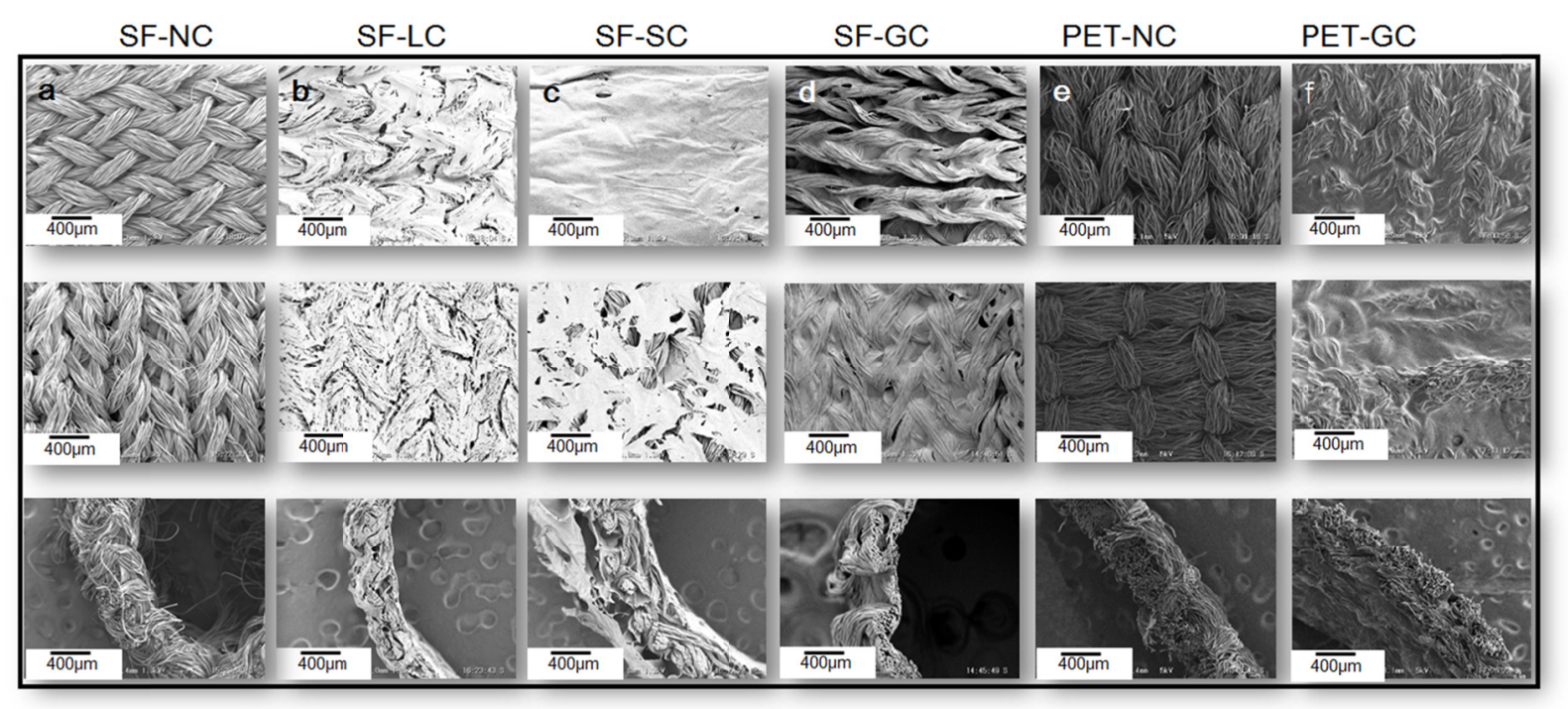

Figure 1. SEM images of the top-down, outer surface, inner surface and cross section of before coating SF and PET grafts (a, e), SF graft with lyophilized fibroin coating (b), SF graft with fibroin sponge coating (c), SF graft with gelatin-coated (d) and PET graft with gelatin coated (f), respectively

Figure 2(a) and (b) show high magnification SEM images of the inner surfaces of SF-GC and PET-GC before blood contact. Both the coated surfaces were smoothly covered with gelatin, although exposed fibres could be observed in a few places because of cracking and peeling of coating materials. It is generally assumed that the results in static state mainly reflect the effect of the material itself, such as the surface charge. In the rotation condition, blood is exposed to shear stress due to the material surface, which has been shown to increase activation of blood components.

Therefore, the results include the effect of surface shape of the coating and weave structure of the artificial blood vessel. Considering these aspects, we decided to perform our experiments in two conditions, static and rotation conditions, in order to determine the hemocompatibility of SF vascular tube and coating materials. Figure 2 (c)-(f) show the inner surfaces of SF-GC and PET-GC after blood contact under static (c, d) and rotation condition (e, f). The blood contact reaction, represented by fibrin deposition, platelet adhesion and platelet aggregation of these grafts, are summarized in Figure 3 top-down static and rotation condition. Blood contact reaction rates were evaluated on a scale of five for each point and compared by one way ANOVA and Tukey's multiple comparison test. Under both condition, the degree and standard deviation of these observations were weaker for SF-GC than for PET-GC. Figure 4 shows SEM observations of SF-LC and SF-SC after blood contact under static or rotation condition.

Those are summarized in Figure 5 together with that of SF-GC. In the case of static condition, there was no apparent difference among the three grafts. In the case of rotation condition, the degree of fibrin deposition, platelet deposition and platelet aggregation on SF-GC were weaker than those on SF-LC or SF-SC. 


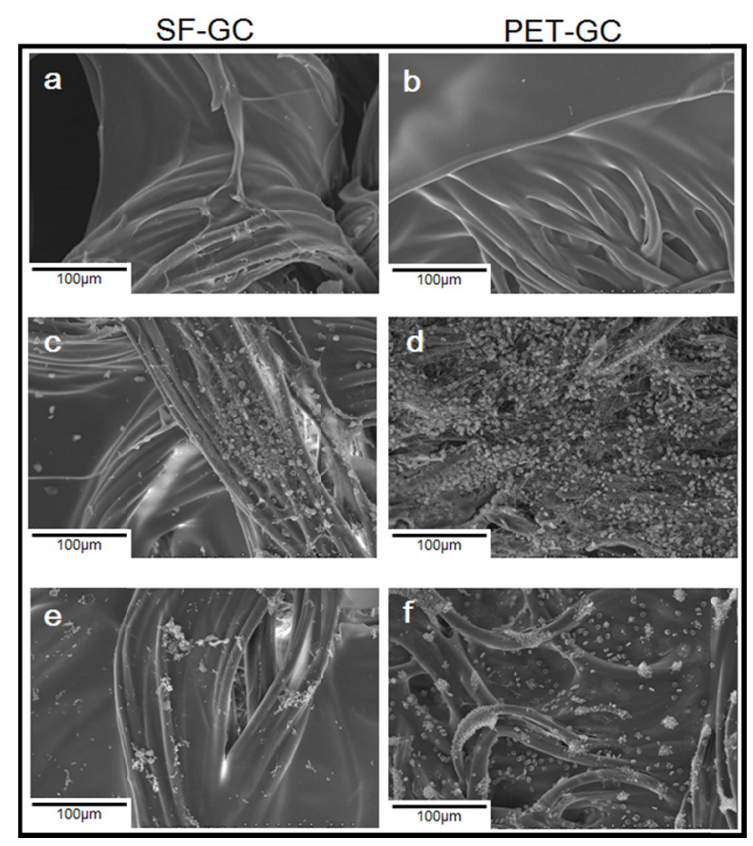

Figure 2. SEM images of the inner surface of gelatin-coated SF grafts (SF-GC; a, c, e) and gelatin-coated PET grafts (PET-GC; $b, d, f)$ before blood contact $(a, b)$ and after blood contact under static (c, d) and rotation conditions $(\mathrm{e}, \mathrm{f})$
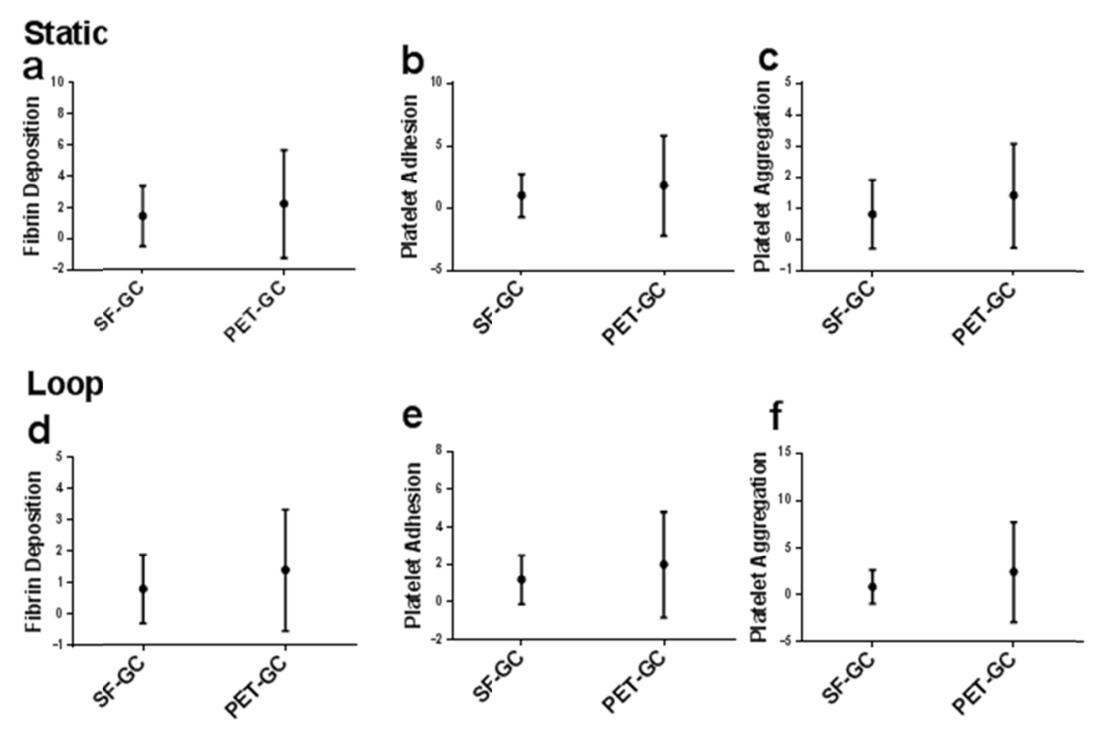

Figure 3. SEM observations of SF-GC and PET-GC graft surfaces under static $(a, b, c)$ and rotation condition $(\mathrm{d}, \mathrm{e}, \mathrm{f})$ to evaluate fibrin deposition, platelet adhesion and platelet aggregation, respectively 


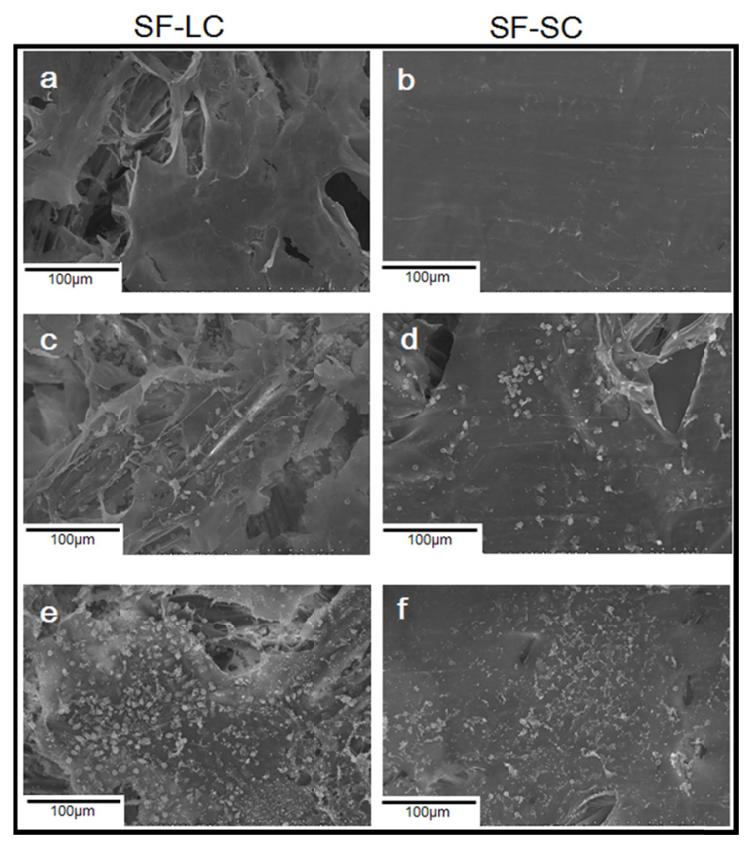

Figure 4. SEM images of the inner surface of SF grafts with lyophilized fibroin coating (SF-LC; a, c, e) and SF grafts with fibroin sponge coating (SF-SC; $b, d, f)$ before blood contact $(a, b)$ and after blood contact under static $(c, d)$ and rotation conditions $(e, f)$
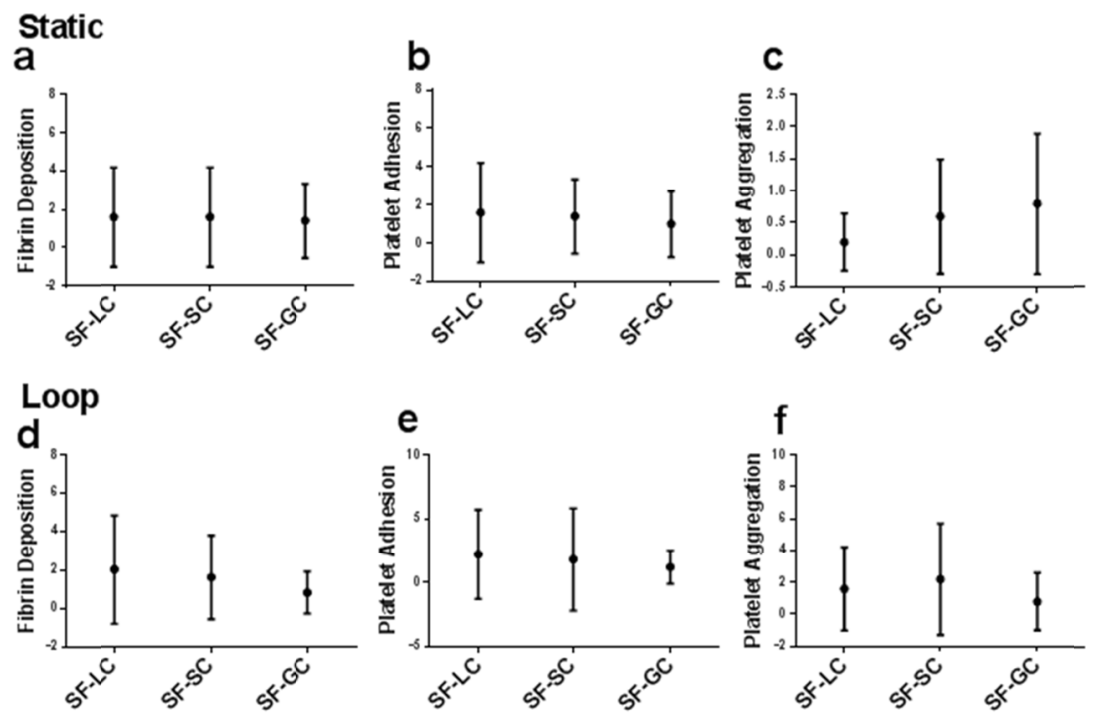

Figure 5. SEM observations of SF-LC, SF-SC and SF-GC graft surfaces under static (a,b,c) and rotation condition $(\mathrm{d}, \mathrm{e}, \mathrm{f})$ to evaluate fibrin deposition, platelet adhesion and platelet aggregation, respectively

\subsection{TAT, $\beta-T G, C 3 a$ and SC5b-9 Evaluation}

The concentrations of TAT, $\beta$-TG, C3a and SC5b-9 in blood, measured after exposure to SF-GC and PET-GC, are summarized in Figure 6. The concentrations of TAT and $\beta$-TG were lower for SF-GC than for PET-GC under static and rotation condition. The PET-GC showed significantly higher TAT concentration compared with positive control of that blank tube either under static or rotation condition. The concentration of C3a with SF-GC was almost the same as that with the blank and PET-GC. However under static condition PET-GC showed significantly higher sC5b-9 concentration than positive control. Interestingly the sC5b-9 concentration of SF-GC under rotation condition was significantly higher than those of PET-GC and positive control. We suggest that 
gelatin released from surface of SF-GC induces increase of the sC5b-9 concentration.

The concentrations of TAT, $\beta$-TG, C3a and sC5b-9 in blood after exposure to SF grafts with different coatings (SF-LC, SF-SC and SF-GC) are summarized in Figure 7. Under static condition, the concentrations of all three SF grafts were very similar except that for the concentration of sC5b-9. SF-SC showed significantly higher sC5b-9 concentration than other graft sample including blank tube. Under rotation condition, the concentrations of TAT and $\beta-T G$ of the SF-LC and SF-SC grafts were higher than those of the blank and SF-GC. However, high concentrations were obtained for SF-LC and SF-SC grafts from the same blood sample, and therefore the sample seems sensitive to SF than the other two blood samples.

a
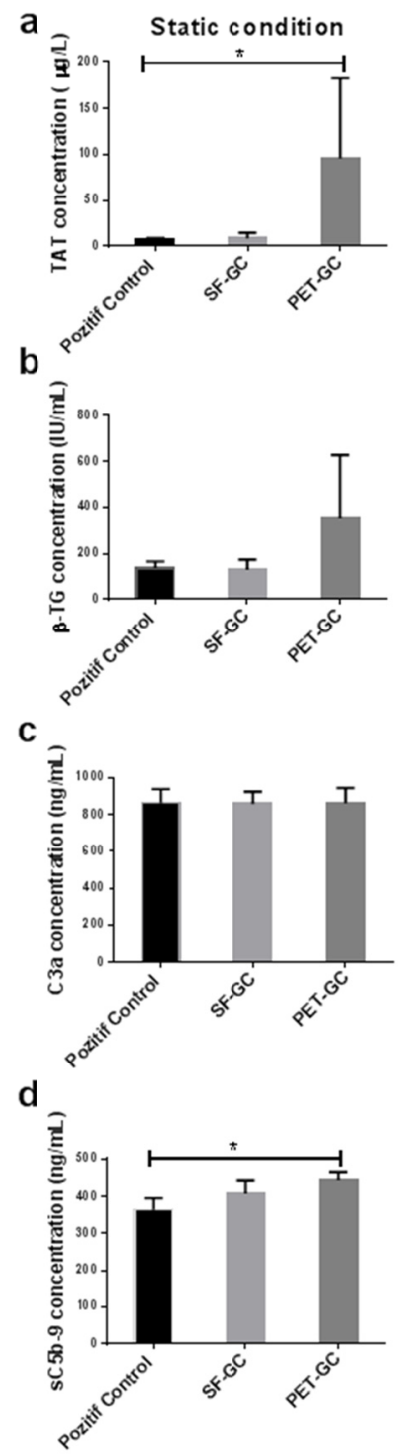

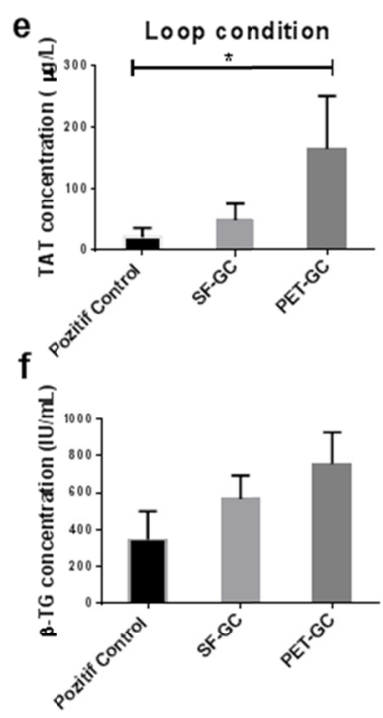

g

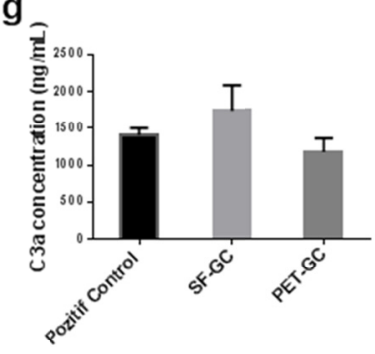

$\mathrm{h}$

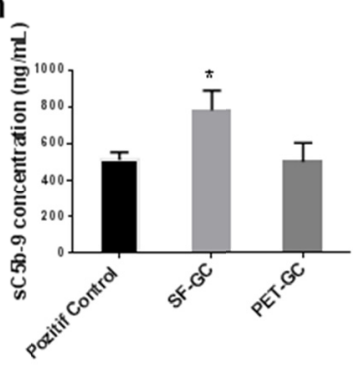

Figure 6. Concentration of TAT (a, e), $\beta$-TG $(b, f), C 3 a(c, g)$ and SC5b-9 (d, h) (top-down) in human whole blood after contact with SF grafts coated with gelatin (SF-GC) and with PET grafts coated with gelatin (PET-GC) under static conditions ( $\mathrm{a}, \mathrm{b}, \mathrm{c}, \mathrm{d}$ ) and rotation conditions $(\mathrm{e}, \mathrm{f}, \mathrm{g}, \mathrm{h}$ ). Values obtained with a blank test (positive control) are also shown as references. [Values are given as mean \pm standard deviation (SD). A P level $<0.05$ was considered statistically significant]

\subsection{Blood Evaluation by Rat Implantation}

Coagulation activity was evaluated by PT and APTT. No difference was observed among the different coating 
materials for either value. PT of the sham-operated rats was $14.3 \pm 1.3 \mathrm{~s}$ and that of the SF-LC, SF-SC and SF-GC rats was reduced to $11.9 \pm 0.4 \mathrm{~s}, 12.0 \pm 0.1 \mathrm{~s}$ and $12.1 \pm 0.3 \mathrm{~s}$, respectively [Figure 7(a)]. Each coating material resulted in almost the same reduction in PT. This tendency was also observed for APTT [Figure 7(b)]. APPT showed greater reduction than PT for all the grafts, except for the sham-operated rats.
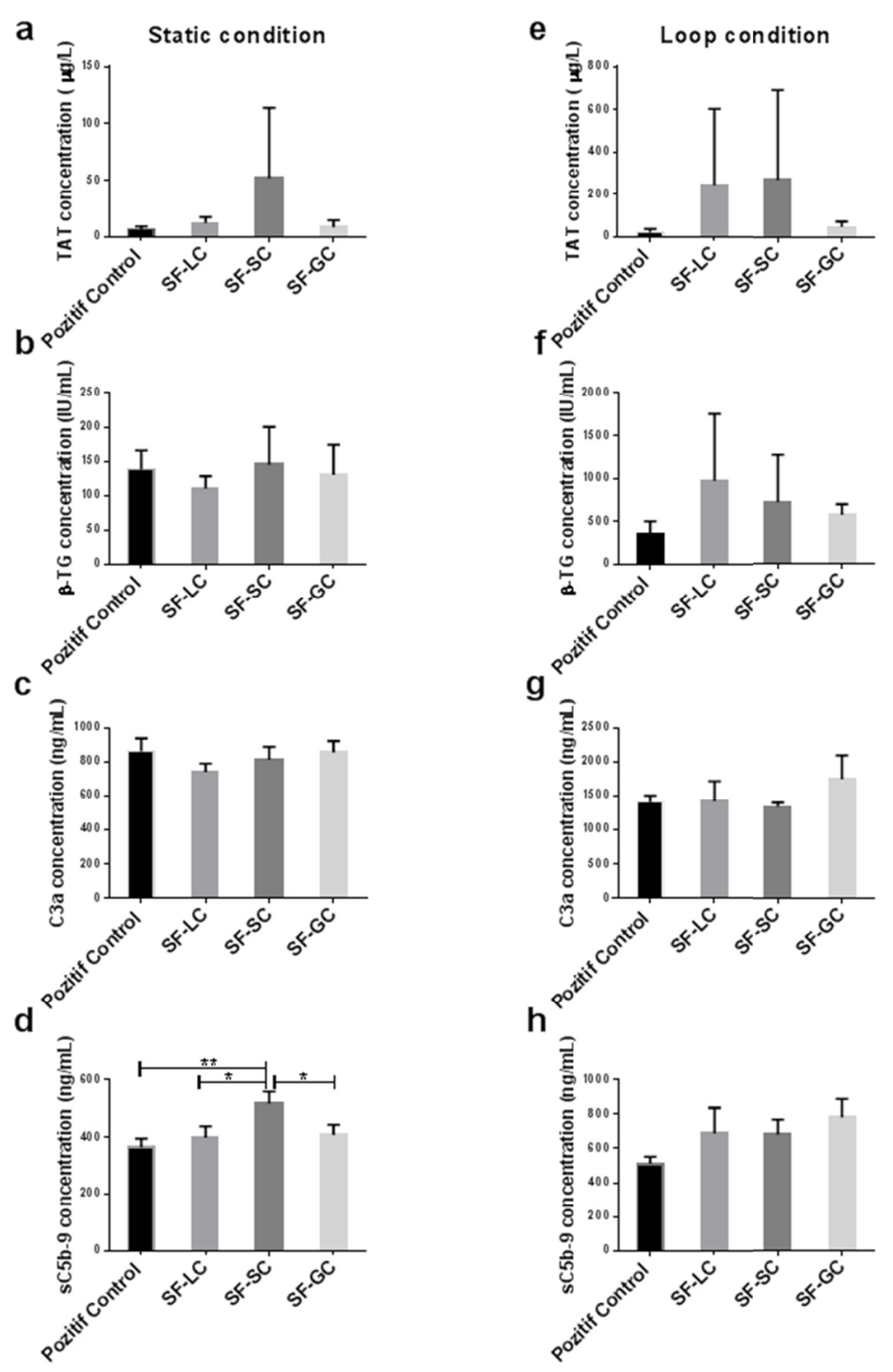

Figure 7. Concentration of TAT (a, e), $\beta$-TG (b, f), C3a (c, g) and SC5b-9 (d, h) (top-down) in human whole blood after contact with SF grafts coated with lypholization (SF-LC), sponge (SF-SP) and gelatin (SF-GC) under static conditions $(\mathrm{a}, \mathrm{b}, \mathrm{c}, \mathrm{d})$ and rotation conditions $(\mathrm{e}, \mathrm{f}, \mathrm{g}, \mathrm{h})$. Values obtained with a blank test (positive control) are also shown as references. [Values are given as mean \pm standard deviation (SD). A P level $<0.05$ was considered statistically significant]

Blood samples of the SF-SC-implanted rats showed increased WBC levels $\left(105.3 \pm 15.9 \times 10^{2} / \mu \mathrm{L}\right)[$ Figure $7(\mathrm{c})]$. In contrast, the results from other blood samples were almost the same as those from the sham-operated rats, at $66.0 \pm 7.8 \times 10^{2} / \mu \mathrm{L}, 57.5 \pm 3.7 \times 10^{2} / \mu \mathrm{L}$ and $54.8 \pm 5.1 \times 10^{2} / \mu \mathrm{L}$, respectively, for the SF-LC grafts, SF-GC grafts and sham-operated controls. We observed a greater increase in PCT levels in blood samples of the rats implanted with SF-LC \{at $0.43 \pm 0.01 \%$ for [Figure 7(e)]\} compared with those in blood samples of the rats implanted with SF-SC and SF-GC and the sham-operated controls $[0.36 \pm 0.02 \%, 0.33 \pm 0.05 \%$ and $0.29 \pm$ 
$0.04 \%$, respectively]. Platelet activities in rat blood in response to coating materials were compared with those of blood from sham-operated rats. Neither the PLT nor PDW value showed any difference when compared with the values in sham-operated animals [Figure 7(d), (f)]. Moreover, it was observed that PLT values of blood samples of the SF-LC- and SF-SC-implanted rats were slightly higher than those of blood samples of the sham-operated rats.

\section{Discussion}

Vascular prostheses should have low thrombogenicity to avoid blood platelet adhesion and thus retain vascular patency and minimize intimal hyperplasia (Bauer et al., 1995). Until date, use of whole blood is the best in vitro assessment available to evaluate overall blood compatibility because studies focusing on either platelet activation or coagulation activation may overlook activating cross-talk between these pathways (Gorbet et al., 2004).
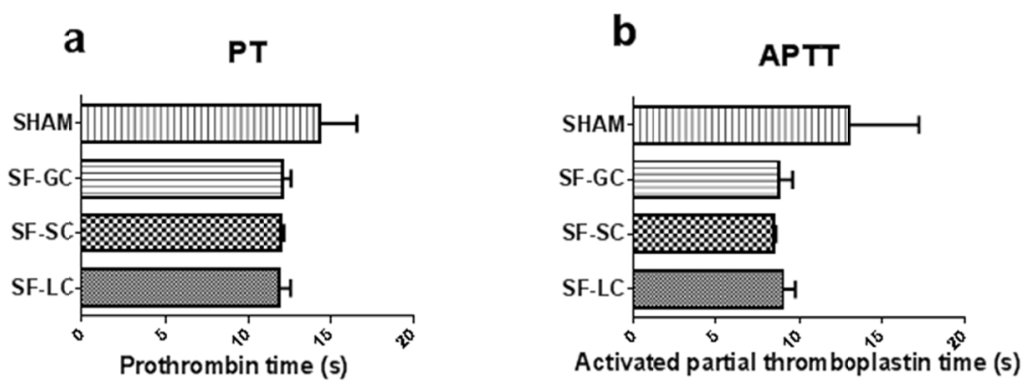

c

WBC

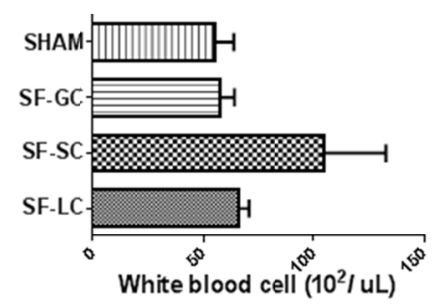

e

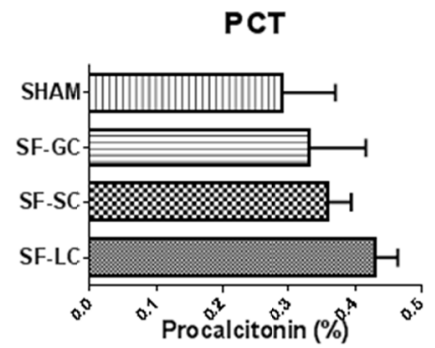

d

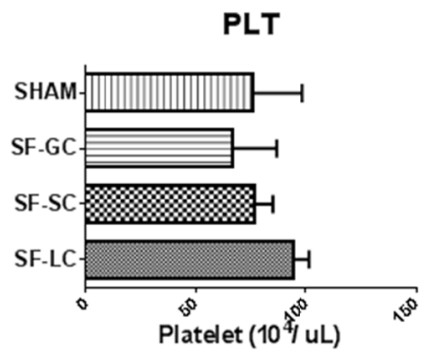

f

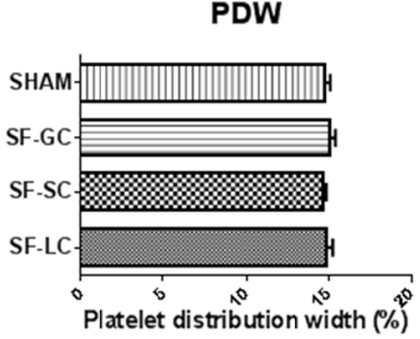

Figure 8. In vivo blood evaluation of SF grafts with various coatings implanted into the rat abdominal aorta.

Evaluated blood values are PT (a), APTT (b), WBC (c), PLT (d), PCT (e) and PDW (f) shown with sham-operated animals as reference. [Values are given as mean \pm standard deviation (SD). A P level $<0.05$ was considered statistically significant] 
In this study, to examine the hemocompatibility of an SF-based artificial vascular graft, thrombogenicity, coagulation activation, platelet and complement system activation were evaluated by human blood contact in vitro with SEM observation and measurements of TAT, $\beta-\mathrm{TG}, \mathrm{C} 3 \mathrm{a}$ and SC5b-9. Activation of the coagulation system, including platelets, plays an important role in the process of thrombus formation. Activation of the coagulation system begins with thrombin generation, which mediates conversion of fibrinogen to fibrin. Formation of TAT complexes following the neutralization of thrombin by anti-thrombin III are used as a marker of thrombin generation. Therefore, the increase in the TAT concentration indicates an increase in thrombin-mediated coagulation. SEM observation of fibrin deposition on the material surface also indicates coagulation progression. Furthermore, primary thrombus formation results from platelet deposition (Blockmans et al., 1995). On a high thrombogenic material surface, platelets adhere and activate with elongation. Next, factors in platelet granules are released, finally leading platelet aggregation. Therefore, SEM observation of platelet adhesion and aggregation on a material surface after blood contact is a reliable method of assessing thrombogenicity. In addition, the aggregation of platelets leads to the release reaction, in which $\alpha$-granules merge with the cell membrane and $\beta$-TG are released to the outside of the cell, together with fibrinogen and platelet factor 4. $\beta$-TG concentration in the blood plasma can thus be an indicator of platelet activation because $\beta$-TG is a specific platelet protein (Chandrasekar et al., 2000). Thus, in in vitro experiments using human whole blood, the thrombogenicity of SF based artificial vascular grafts can be evaluated by TAT, $\beta$-TG, and SEM observation. The foreign surface triggers the activation of the complement systems of the blood, leading to tissue injury and inflammation. C3a anaphylatoxin is produced in the early stages of complement activation. In contrast, SC5b-9 is a substance released from the final product of complement activation. Both C3a and SC5b-9 are important factors for evaluating hemocompatibility because they are released when the complement system is activated following blood contact with foreign materials. Here we examine the hemocompatibility of coating treatment on SF-based artificial vascular grafts using these evaluation endpoints.

First, the hemocompatibility of gelatin-coated SF grafts was evaluated by comparing them with commercially available artificial vascular grafts, which are made of PET with a gelatin coating. SF-GC exhibited weaker thrombus levels, indicated by SEM observation and lower concentrations of TAT and $\beta$-TG after blood contact, compared with PET-GC in both static and rotation conditions. These results indicate that the thrombogenicity of SF-GC is low. In addition, the C3a and SC5b-9 concentrations following static condition exposure to SF-GC were almost the same as the blank levels, showing that this material has little effect on the complement system, similar to PET-GC. These results therefore demonstrate that hemocompatibility of SF-GC is equivalent to or greater than that of PET-GC.

Recent studies, including our group have been manufactured SF vascular grafts for revascularization by different methods. For this purpose the silk micro-tubes prepared by using the layer-by-layer deposition of concentrated SF solution. However, the burst strengths of silk micro-tubes were significantly low and most tubes failed at pressures above the approximate physiological pressures of 100-140 $\mathrm{mm} \mathrm{Hg}$ (coronary artery during systole) or $15-40 \mathrm{~mm} \mathrm{Hg}$ (capillary blood pressure), indicating the need in vivo studies in future (Lovett et al., 2007). Afterward SF solution successfully electrospun into tubular structures with inner diameters of approximately 3 $\mathrm{mm}$ and an average wall thickness of $0.15 \mathrm{~mm}$, by which an average ultimate tensile strength (UTS) of $2.42 \pm$ $0.48 \mathrm{MPa}$ and a linear modulus of $2.45 \pm 0.47 \mathrm{MPa}$ were obtained (Soffer et al., 2008). However Sato et al. obtained electrospun silk tubes with a higher longitudinal UTS and linear modulus of $2.76 \pm 0.13 \mathrm{MPa}$ and 4.94 $\pm 0.16 \mathrm{MPa}$, respectively, which were improved to $3.83 \pm 0.48 \mathrm{MPa}$ and $6.63 \pm 0.77 \mathrm{MPa}$ by silk sponge coating with a composite of PEGDE (Sato et al., 2010). These results were comparable to those from a previous study of silk electrospun mats reported by Ayutsede et al. The average burst strength of the tubular scaffolds was $811 \mathrm{~mm}$ $\mathrm{Hg}$, which is larger than those from graft scaffolds prepared with collagen $(71 \mathrm{~mm} \mathrm{Hg}$ ) or other commonly used natural biomaterials (Ayutsede et al., 2005). However, further development is required for spinning material to reach the gold standard of the saphenous vein, of which the burst strength is $1800 \mathrm{mmHg}$ (Nishibe et al., 2007). Meanwhile, evaluation of the biological potential of electrospun silk matrices for vascular tissue engineering was determined by HAECs and HCASMCs. Good retention of vascular cells was demonstrated; cell numbers remained high for HCASMCs over 36 days and HAECs over 14 days in culture with high seeding density. Positive staining of smooth muscle $\alpha$-actin ( $\alpha$-SMA) and smooth muscle myosin heavy chain 2 (SM-MHC2) for HCASMCs, as well as CD146, VE-cadherin, and PECAM-1 for HAECs, was identified. Furthermore, expression of the major extracellular matrix (ECM) components of vessel walls (collagen I and elastin) was demonstrated at both protein and transcript levels for HCASMCs, which indicated the SMC functionality on electrospun silk matrices (Zhang et al., 2008).

Seib et al. previously reported evaluation of chronic hemocompatibility of SF films and comparison with 
clinically relevant reference materials (Seib et al., 2012). In their study, the complement response to SF represented a similar percentage of polylactide-co-glycolide films and initials under static conditions. However it is difficult to implement such films to use as a coating material. Also our previous results showed long patency period for SF knitted vascular prosthesis on rat implantation study, however dog implantation results did not showed same endothelialisation and tissue formation. Our results show a slightly better complement response to SF grafts than to PET grafts. In addition, we observed lower coagulation (TAT) and platelet ( $\beta$-TG) activation than that of silk films reported in that study.

Finally, the blood contact reaction among SF-LC, SF-SC and SF-GC grafts were evaluated to examine the effect of coating materials with respect to subacute hemocompatibility of SF grafts. Complement system activation as a measure of the inflammatory reaction of human blood was equivalent for all the coating materials, but in the in vivo rat study, SF-SC showed a slight increase in WBC levels compared with the other coating types, although this was not a significant difference. Moreover, PCT levels did not support this increase. We also evaluated PLT for each coating because platelets have an important role in haemostasis, which preserves the integrity of the vascular wall through formation of the platelet plug. Platelets are activated when they contact any thrombogenic surfaces, such as injured endothelium, subendothelium or artificial surfaces (Bittl et al., 1996; Sperling et al., 2009). PLT showed the same tendency under static conditions of $\beta$-TG levels for human blood. All samples showed almost the same reduction in PT and APTT; the sham-operation time for both values was also reduced. Normal rat PT and APTT values were reported to be $15.9 \pm 2.3 \mathrm{~s}$ and $22.1 \pm 2.8 \mathrm{~s}$, respectively (Kurata et al., 2003). The present results support the theory that vascular injury is associated with reduced PT and APTT in rats. However, no correlation between reduction and coating types was observed. According to these results, SF grafts have good hemocompatibility because all blood samples from the coated SF grafts showed very similar values for all the parameters evaluated in this study, with the sham-operated rats as the positive control.

Clinical manifestations of haemoincompatibility are acute and subacute thrombotic occlusion in medium sized grafts (4-6 mm) and acute failure of small diameter vascular grafts (Sperling et al., 2009). In our previous studies, we prepared SF grafts by braiding, flattening and winding the SF fibres onto a cylindrical polymer tube, followed by coating with an aqueous SF solution (Enomoto et al., 2010). The grafts, which were $1.5 \mathrm{~mm}$ in inner diameter and $10 \mathrm{~mm}$ in length, as used in the present study, were implanted into the rat abdominal aorta. An excellent patency rate $(85.1 \%, \mathrm{n}=27, \mathrm{P}<0.01)$ was obtained at 1 year after grafting with the SF fibres. This patency rate was remarkably higher than that of the PTFE grafts $(30 \%, n=10)$. Endothelial and smooth muscle cells rapidly became organized to form the inner layer of the SF grafts. Sirius Red staining revealed that the collagen content of the fibroin grafts significantly increased 1 year after implantation, with a decrease in the SF content (Enomoto et al., 2010). In this study, SF-based vascular grafts showed better compatibility with gelatin coating (SF-GC) than PET grafts (PET-GC). In addition, SF coating methods (SF-LC, SF-SC) showed almost the same compatibility as gelatin coating (SF-GC). Our previous results (Aytemiz et al., 2012) and the results of in vitro evaluation of the hemocompatibility of the SF grafts show that SF materials have the potential to replace currently used commercial PET grafts and PTFE, if the success rate of in vivo studies on large animals can be increased by enhancing the mechanical properties of the grafts.

We investigated the compatibility of SF materials with whole human blood. We quantified the inflammatory and haemostasis response at the cellular and humoral levels. In addition, we reinforced the results with SEM imaging for each blood sample. We also compared the SF vascular tube materials with PET vascular prosthetic grafts currently in use. Our results support the notion that $\mathrm{SF}$ has potential as a material for future vascular regeneration throughout the post-transplantation period.

\section{Acknowledgements}

TA acknowledges the support from Ministry of Agriculture, Forestry and Fisheries of Japan (Agri-Health Translational Research Project) and from Grant-in-Aid for Scientific Research from Ministry of Education, Science, Culture and Supports of Japan (23245045). YS acknowledges support from Women's Future Development Organization in Tokyo University of Agriculture and Technology.

\section{References}

Asakura, T., \& Kaplan, D. L. (1994). Silk Production and Processing. In C. J. Arutzen (Ed.), Encyclopedia of Agricultural Science (pp. 1405-1408). Academic Press.

Aytemiz, D., Sakiyama, W., Suzuki, Y., Nakaizumi, N., Tanaka, R., Ogawa, Y., ... Asakura, T. (2012). Small-diameter knitted silk vascular grafts ( $3 \mathrm{~mm}$ diameter) with a double-raschel knitted silk tube coated by silk fibroin sponge. Adv. Healthcare Mater, 5, 2192-2200. 
Ayutsede, J., Gandhi, M., Sukigara, S., Micklus, M., Chen, H., \& Ko, F. (2005). Regeneration of Bombyx mori silk by electrospinning. Part 3: characterization of electrospun nonwoven mat. Polymer, 46, 1625-1634. http://dx.doi.org/10.1016/j.polymer.2004.11.029

Bauer, K. A., \& Rosenberg, R. D. (1995). Control of coagulation factors. In E. Beutler, M. A. Lichtman, B. S. Coller, \& T. J. Kipps (Eds.), Williams hematology (5th ed., pp. 1239-50). New York: McGraw-Hill.

Bittl, J. A. (1996). Coronary stent occlusion: thrombus horribilis. J. Am. Coll. Cardiol., 28, 368-70. http://dx.doi.org/10.1016/0735-1097(96)00183-0

Blockmans, D., Deckmyn, H., \& Vermylen, J. (1995). Platelet activation. Blood Rev., 9, 143-56. http://dx.doi.org/10.1016/0268-960X(95)90020-9

Bondar, B., Fuchs, S., Motta, A., Migliaresi, C., \& Kirkpatrick, C. J. (2008). Functionality of endothelial cells on silk fibroin nets: comparative study of micro- and nanometric fibre size. Biomaterials, 29, 561-572. http://dx.doi.org/10.1016/j.biomaterials.2007.10.002

Chandrasekar, B., \& Tanguay, J. F. (2000). Platelets and restenosis. J. Am. Coll. Cardiol., 35, 555-62. http://dx.doi.org/10.1016/S0735-1097(99)00596-3

Demura, M., \& Asakura, T. (1989a). Immobilization of Glucose-Oxidase with Bombyx-Mori Silk Fibroin by Only Stretching Treatment and Its Application to Glucose Sensor. Biotechnol. Bioeng, 33, 598-603. http://dx.doi.org/10.1002/bit.260330513

Demura, M., \& Asakura, T. (1991). Porous Membrane of Bombyx-Mori Silk Fibroin - Structure Characterization, Physical-Properties and Application to Glucose-Oxidase Immobilization. J. Membrane Sci., 59, 39-52. http://dx.doi.org/10.1016/S0376-7388(00)81220-X

Demura, M., Asakura, T., \& Kuroo, T. (1989). Immobilization of biocatalysts with Bombyx mori silk fibroin by several kinds of physical treatment and its application to glucose sensors. Biosensors, 4, 361-72. http://dx.doi.org/10.1016/0265-928X(89)80002-1

Devine, C., \& McCollum, C. (2004). Heparin-bonded Dacron or polytetrafluorethylene for femoropopliteal bypass: five-year results of a prospective randomized multicenter clinical trial. J. Vasc. Surg., 40, 924-31. http://dx.doi.org/10.1016/j.jvs.2004.08.033

Enomoto, S., Sumi, M., Kajimoto, K., Nakazawa, Y., Takahashi, R., Takabayashi, C., ... Sata, M. (2010). Long-term patency of small-diameter vascular graft made from fibroin, a silk-based biodegradable material. J. Vasc. Surg., 51, 155-64. http://dx.doi.org/10.1016/j.jvs.2009.09.005

Gorbet, M. B., \& Sefton, M. V. (2004). Biomaterial-associated thrombosis: roles of coagulation factors, $\begin{array}{llll}\text { complement, platelets and leukocytes. } & \text { Biomaterials, 25, }\end{array}$ http://dx.doi.org/10.1016/j.biomaterials.2004.01.023

Gupta, V., Davis, G., Gordon, A., Altman, A. M., Reece, G. P., Gascoyne, P. R., \& Mathur, A. B. (2010). Endothelial and stem cell interactions on dielectrophoretically aligned fibrous silk fibroin-chitosan scaffolds. J. Biomed. Mater. Res. A., 94, 515-523.

Heyligers, J. M. M., Verhagen, H. J. M., Rotmans, J. I., Weeterings, C., de Groot, P. G., Moll, F. L., \& Lisman, T. (2006). Heparin immobilization reduces thrombogenicity of small-caliber expanded polytetrafluoroethylene grafts. J. Vasc. Surg., 43, 587-91. http://dx.doi.org/10.1016/j.jvs.2005.10.038

How, T. V., Guidoin, R., \& Young, S. K. (1992). Engineering design of vascular prostheses. Proc Inst Mech Eng H., 206, 61-71. http://dx.doi.org/10.1243/PIME_PROC_1992_206_269_02

Huang, F., Sun, L., \& Zheng, J. (2008). In vitro and in vivo characterization of a silk fibroin-coated polyester vascular prosthesis. Artif Organs, 32, 932-41. http://dx.doi.org/10.1111/j.1525-1594.2008.00655.x

Jin, H. J., Chen, J. S., Karageorgiou, V., Altman, G. H., \& Kaplan, D. L. (2004). Human bone marrow stromal cell responses on electrospun silk fibroin mats. Biomaterials, 25, 1039-47. http://dx.doi.org/10.1016/S0142-9612(03)00609-4

Kurata, M., Sasayama, Y., Yamasaki, N., Kitazawa, I., Hamada, Y., \& Hoorii, I. (2003). Mechanism for shortening PT and APTT in dogs and rats -effect of fibrinogen on PT and APTT. J. Toxicol. Sci., 28, 439-43. http://dx.doi.org/10.2131/jts.28.439

Liu, S., Dong, C., Lu, G., Lu, Q., Li, Z., Kaplan, D. L., \& Zhu, H. (2013). Bilayered vascular grafts based on silk proteins. Acta Biomater, 9, 8991-9003. http://dx.doi.org/10.1016/j.actbio.2013.06.045 
Lovett, M., Cannizzaro, C., Daheron, L., Messmer, B., Vunjak-Novakovic, G., \& Kaplan, D. L. (2007) Silk fibroin microtubes for blood vessel engineering. Biomaterials, 28, 5271-5279. http://dx.doi.org/10.1016/j.biomaterials.2007.08.008

Lovett, M., Cannizzaro, C. M., Vunjak-Novakovic, G., \& Kaplan, D. L. (2008). Gel spinning of silk tubes for tissue engineering. Biomaterials, 29, 4650-7. http://dx.doi.org/10.1016/j.biomaterials.2008.08.025

Lovett, M., Eng, G., Kluge, J. A., Cannizzaro, C., Vunjak-Novakovic, G., \& Kaplan, D. L. (2010). Tubular silk

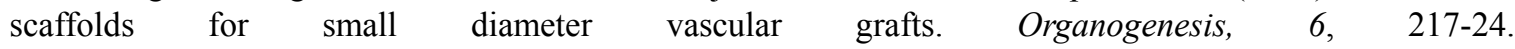
http://dx.doi.org/10.4161/org.6.4.13407

Murugesan, S., Xie, J., \& Linhardt, R. J. (2008). Immobilization of heparin: approaches and applications. Curr. Top. Med. Chem., 8, 80-100. http://dx.doi.org/10.2174/156802608783378891

Nishibe, T., Kondo, Y., Muto, A., \& Dardik, A. (2007). Optimal prosthetic graft design for small diameter vascular grafts. Vascular, 15, 356-60. http://dx.doi.org/10.2310/6670.2007.00053

Rockwood, D. N., Gil, E. S., Park, S. H., Kluge, J. A., Grayson, W., Bhumiratana, S., ... Kaplan, D. L. (2011). Ingrowth of human mesenchymal stem cells into porous silk particle reinforced silk composite scaffolds: An in vitro study. Acta. Biomater, 7, 144-51. http://dx.doi.org/10.1016/j.actbio.2010.07.020

Seib, F. P., Maitz, M. F., Hu, X. A., Werner, C., \& Kaplan, D. L. (2012). Impact of processing parameters on the haemocompatibility of Bombyx mori silk films. Biomaterials, 33, 1017-23. http://dx.doi.org/10.1016/j.biomaterials.2011.10.063

Sato, M., Nakazawa, Y., Takahashi, R., Tanaka, K., Sata, M., Aytemiz, D., \& Asakura, T. (2010). Small-diameter vascular grafts of Bombyx mori silk fibroin prepared by a combination of electrospinning and sponge coating. Mater. Lett, 64, 1786-1788.

Soffer, L., Wang, X. Y., Mang, X. H., Kluge, J., Dorfmann, L., Kaplan, D. L., \& Leisk, G. (2008) Silk-based electrospun tubular scaffolds for tissue-engineered vascular grafts. J. Biomat. Sci-Polym. E., 19, 653-64.

Sperling, C., Fischer, M., Maitz, M. F., \& Werner, C. (2009). Blood coagulation on biomaterials requires the combination of distinct activation processes. Biomaterials, 30, 4447-56. http://dx.doi.org/10.1016/j.biomaterials.2009.05.044

Stoppato, M., Stevens, H. Y., Carletti, E., Migliaresi, C., Motta, A., \& Guldberg, R. E. (2013). Effects of silk fibroin fiber incorporation on mechanical properties, endothelial cell colonization and vascularization of PDLLA scaffolds. Biomaterials, 34, 4573-4581. http://dx.doi.org/10.1016/j.biomaterials.2013.02.009

Tepe, G., Schmehl, J., Wendel, H. P., Schaffner, S., Heller, S., Gianotti, M., Claussen, C. D., \& Duda, S. H. (2006). Reduced thrombogenicity of nitinol stents--in vitro evaluation of different surface modifications and coatings. Biomaterials, 27, 643-50. http://dx.doi.org/10.1016/j.biomaterials.2005.06.004

Vepari, C., \& Kaplan, D. L. (2007). Silk as a Biomaterial. Prog. Polym. Sci., 32, 991-1007. http://dx.doi.org/10.1016/j.progpolymsci.2007.05.013

Zhang, X. H., Baughman, C. B., \& Kaplan, D. L. (2008). In vitro evaluation of electrospun silk fibroin scaffolds for vascular cell growth. Biomaterials, 29, 2217-27. http://dx.doi.org/10.1016/j.biomaterials.2008.01.022

\section{Copyrights}

Copyright for this article is retained by the author(s), with first publication rights granted to the journal.

This is an open-access article distributed under the terms and conditions of the Creative Commons Attribution license (http://creativecommons.org/licenses/by/3.0/). 